\title{
Density measurements using the Background Oriented Schlieren technique
}

\author{
L. Venkatakrishnan, G. E. A. Meier
}

\begin{abstract}
This paper describes the implementation of a novel technique called Background Oriented Schlieren that can produce quantitative visualization of density in a flow. This technique uses only a digital still camera, a structured background, and inverse tomographic algorithms which can extract two-dimensional slices from a three-dimensional flow. This has been applied to obtain the density field for an axisymmetric supersonic flow over a conecylinder model. Comparisons with cone tables show excellent agreement.
\end{abstract}

\section{List of symbols}

$\begin{array}{ll}\beta & \text { wave angle } \\ \theta & \text { viewing angle } \\ \rho & \text { density }\left(\mathrm{kg} / \mathrm{m}^{3}\right) \\ \lambda & \text { wavelength }(\mathrm{m}) \\ \varepsilon & \text { angle of deflection } \\ D & \text { diameter of cylinder } \\ f & \text { focal length of imaging lens } \\ G(\lambda) & \text { Gladstone-Dale number } \\ j & \sqrt{-1} \\ n & \text { refractive index } \\ M & \text { Mach number } \\ P & \text { projection } \\ S & \text { surface } \\ x & \text { horizontal distance (along axis of symmetry) } \\ y & \text { vertical distance } \\ \Delta y & \text { image displacement }\end{array}$

Received: 31 March 2003 / Accepted: 1 March 2004

Published online: 8 April 2004

(C) Springer-Verlag 2004

L. Venkatakrishnan ( $\square)$

Experimental Aerodynamics Division,

National Aerospace Laboratories,

Bangalore, India

E-mail: venkat@ead.cmmacs.ernet.in

Tel.: +91-80-5223942

Fax: +91-80-5051566

G. E. A. Meier

DLR Institute of Fluid Mechanics,

Göttingen, Germany

This work was supported by a grant from the Aeronautical Research and Development Board Government of India. The technical support of the NAL $0.3 \mathrm{~m}$ trisonic wind tunnel staff during the imaging is acknowledged. The authors wish to thank the two anonymous referees who helped make the methodology clearer to the reader and user, and the referee who drew the attention to earlier work and current effort along similar lines.
$\Delta y^{\prime} \quad$ virtual image displacement

$\Delta Z_{\mathrm{D}} \quad$ half-width of region of disturbance

$Z$

horizontal distance (origin on background plane)

$\begin{array}{ll}\text { Subscripts } \\ \infty & \text { freestream conditions } \\ \text { B } & \text { background } \\ \text { D } & \text { dot pattern } \\ \text { i } & \text { image } \\ \text { S } & \text { surface }\end{array}$

1

\section{Introduction}

The development of new flow diagnostic tools that are nonintrusive and quantitative, as well as applicable to real life full-scale flows, is an important area of experimental aerodynamics research. There is considerable research activity underway aimed at integrating new technological developments in various fields and at evolving new techniques of flow quantification. While all optical techniques used to study density fields in transparent media (usually gases or liquids) depend on variation of the index of refraction in the medium and the resulting effects on a light beam passing through the test region, quite different quantities are measured with each one. Techniques like Schlieren and shadowgraph provide qualitative information on the first and second derivatives of density respectively. Interferometry, on the other hand, provides quantitative information about the density field, but setting up such instrumentation requires tremendous effort and care.

The choice of lasers as a source of illumination, improvements in CCD technology, and fast computers for image acquisition and analysis have resulted in a resurgence of quantitative optical methods. In recent years, there have been several attempts to quantify certain flow features such as the amplitude of internal waves (Sutherland et al 1999; Onu et al 2003), density gradients (McMackin et al 1995; Meier 1999) and density perturbations (Dalziel et al 1998, 2000), broadly using the Schlieren principle. Some of the common steps in such procedures include: imaging (with a CCD camera) of a background through a flow of interest, and software analysis that manipulates the data to find the density or density gradient field. McMackin et al (1995) used a lenslet array (Hartmann sensor) to split a single laser beam into a number of sub-apertures and then detect their individual 
focal spot intensity pattern changes which occur due to the optical path differentials of the phase object. Thereafter tomographic techniques were used to reconstruct the three-dimensional density gradient field. A method called "synthetic Schlieren", proposed by Dalziel et al (1998, 2000), was applied to measure the gradient of the perturbation density and the amplitudes of waves generated by an oscillating cylinder in a stratified flow, and the results agree very well with theoretical values (Dalziel et al 2000; Sutherland et al 1999). Recently, Onu et al (2003) adapted synthetic Schlieren to measure the amplitude of axisymmetric internal waves generated by an oscillating sphere and compare it with vertical displacements obtained from a conductivity probe. They used a simple inverse tomographic technique to measure wave amplitude in the plane of interest, in contrast to the filtered back projection technique (explained later) used here.

In 1999, Meier proposed the Background Oriented Schlieren (BOS) technique, which has the potential to determine the density field. BOS can be described as a simple Schlieren technique based on image displacements of some photographic background by Schlieren in the transfer channel (Meier 1999). A major advantage of this technique is that it requires only a digital still camera with adequate resolution. The principle of Background Oriented Schlieren has broad similarities with synthetic Schlieren; they both exploit the bending of light rays passing through a region of density gradient, followed by mathematical techniques to extract the density field for the plane of interest.

Initial studies (Richard et al 2000; Raffel et al 2000; Meier 2002), demonstrated several possible applications of BOS; these include density fields of helicopter-generated vortices and supersonic jets, but these studies have been predominantly qualitative with no comparisons with theory or measurements.

This paper is an attempt to validate density measurements using the BOS technique for a relatively simple flow at high speeds. The method has been applied to a cone-cylinder flow at Mach 2.0; this model configuration was chosen so that BOS measurements could be compared with results available in cone tables (Sims 1964). The determination of the density field involves the following steps: a) calculation of displacements in the background, which is imaged through the flow of interest, and these displacements are the vectors of density gradient at each point; b) calculation of the line-of-sight integrated density field by solution of the Poisson equation, which is the gradient of the above displacement, and; c) use of the filtered back-projection technique to determine the density field in the actual plane of interest.

An excellent correlation between the density field obtained by BOS and data from cone tables has been found. The usefulness of FBPT for non-axisymmetric flows is also discussed.

\section{2}

\section{Background Oriented Schlieren}

The image of an object is the convolution of the object function and the transfer channel function. Therefore, deconvolution will describe the transfer channel function, if the object and image are given.

Two images of a deliberately structured background are obtained. The first is through the undisturbed transfer channel and the second is through the phase object of interest. The "difference" between these two images provides information about the phase object. The field gradients in the path of the imaging rays cause deflection of the light rays, leading to shifts in the image details.

The principle of the technique is the refractive index variation due to density gradients. This relation between the refractive index $n$ and the density $\rho$ is given by the Gladstone-Dale equation (Merzkirch 1987; Goldstein 1983):

$\frac{n-1}{\rho}=G(\lambda)$

where $G(\lambda)$ is called the Gladstone-Dale constant and depends on characteristics of the gas (medium) and weakly on the frequency of light used (Merzkirch 1987). Values for $G(\lambda)$ can be found from handbooks of physical chemistry. Here, a value of $0.23 \times 10^{-3} \mathrm{~m}^{3} / \mathrm{kg}$ has been used.

In the first step (Meier 1999) an image is taken of a random dot pattern with the air (phase object) at rest. The second image is taken in the presence of the flow containing density gradients. Cross-correlation of the two images yields the displacements of the particles in the $x$ and $y$ directions. Here, $x$ is along the freestream direction - also the axis of symmetry for the present case - (with the origin at the model tip) and perpendicular to the line-ofsight direction $(z)$, and $y$ is the vertical axis (origin at model centerline).

Since the deflection of a single beam contains information about the spatial gradient of the refractive index integrated along the axial path, the image deflection $\varepsilon$ is defined as

$\varepsilon=\frac{1}{n_{0}} \int_{Z_{\mathrm{D}}-\Delta Z_{\mathrm{D}}}^{Z_{\mathrm{D}}+\Delta Z_{\mathrm{D}}} \frac{\delta n}{\delta y} d z$

with the assumption that the half-width of the region of density gradient $\Delta Z_{\mathrm{D}} \ll Z_{\mathrm{D}}$.

Further, it is seen from geometry (see Fig. 1) that the virtual image displacement $\Delta y^{\prime}$ is related to the image displacement $\Delta y$ by the lens distance from the background $Z_{\mathrm{B}}$ and the image distance from the lens $Z_{\mathrm{i}}$, which can be replaced for large $Z_{\mathrm{B}}$ by the focal length $f$ of the lens (Meier 2002):

$\frac{\Delta y^{\prime}}{Z_{\mathrm{B}}}=\frac{\Delta y}{Z_{\mathrm{i}}}=\frac{\Delta y}{f}$

For small deflection angles, $\varepsilon$ can be approximated as

$\varepsilon=\frac{\Delta y^{\prime}}{Z_{\mathrm{D}}}$

hence,

$\varepsilon=\frac{\Delta y Z_{\mathrm{B}}}{Z_{\mathrm{D}} f}$

From Eqs. 2 and 3 it follows that for a given flow field, the shift of the background and the sensitivity 


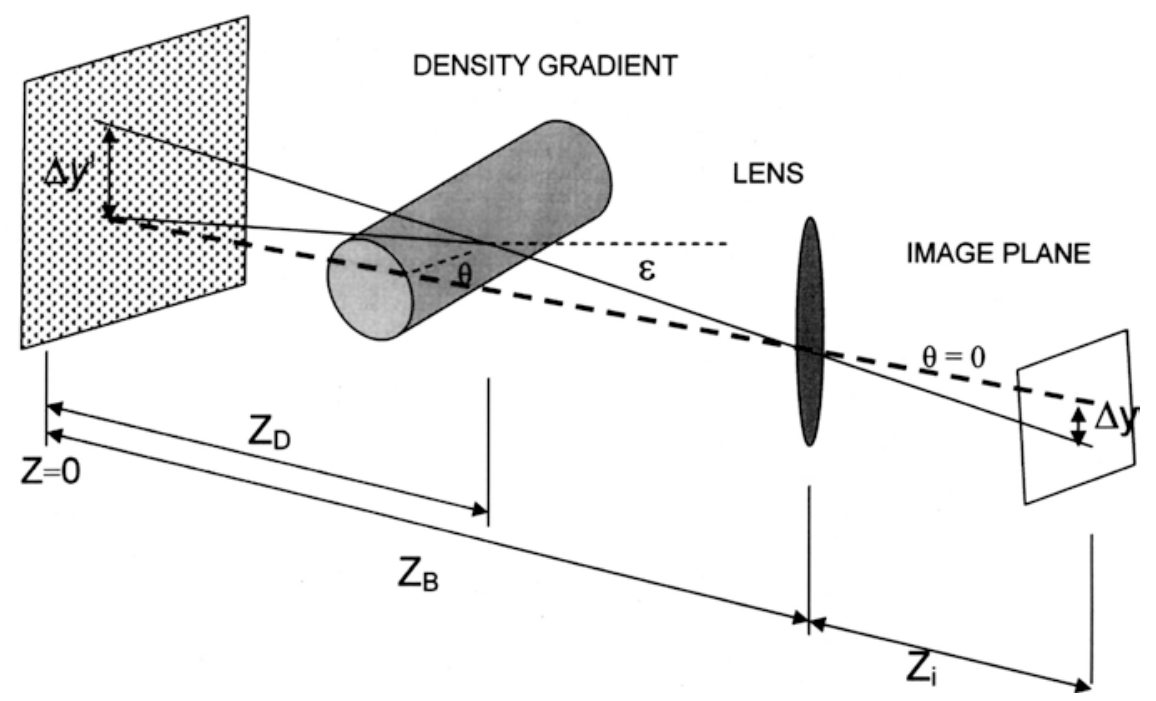

Fig. 1. Optical path for density gradient measurements by light deflection (Meier 1999)
$\left.\left(\left(\frac{\Delta y}{\operatorname{grad} n}\right) \sim Z_{\mathrm{D}} Z_{\mathrm{B}} f\right)\right)$ increases with increasing distance of the background from the density gradient. Increasing focal length $f$ is compensated for by increasing the distance between the camera and the background.

It therefore follows that the obtained displacements are the density gradients at each point in the field. The gradients of this displacement field yield an elliptic partial differential equation also known as the Poisson equation (see Eq. 17), the solution of which yields the line-of-sight integrated density distribution, which is a projection of the three-dimensional density field in the direction of viewing. The distribution of density in a given plane is then calculated by means of a transformation from this projection.

\section{3}

Filtered back projection technique (FBPT)

When a test field cannot be approximated as being infinite in the line-of-sight direction, a mathematical method

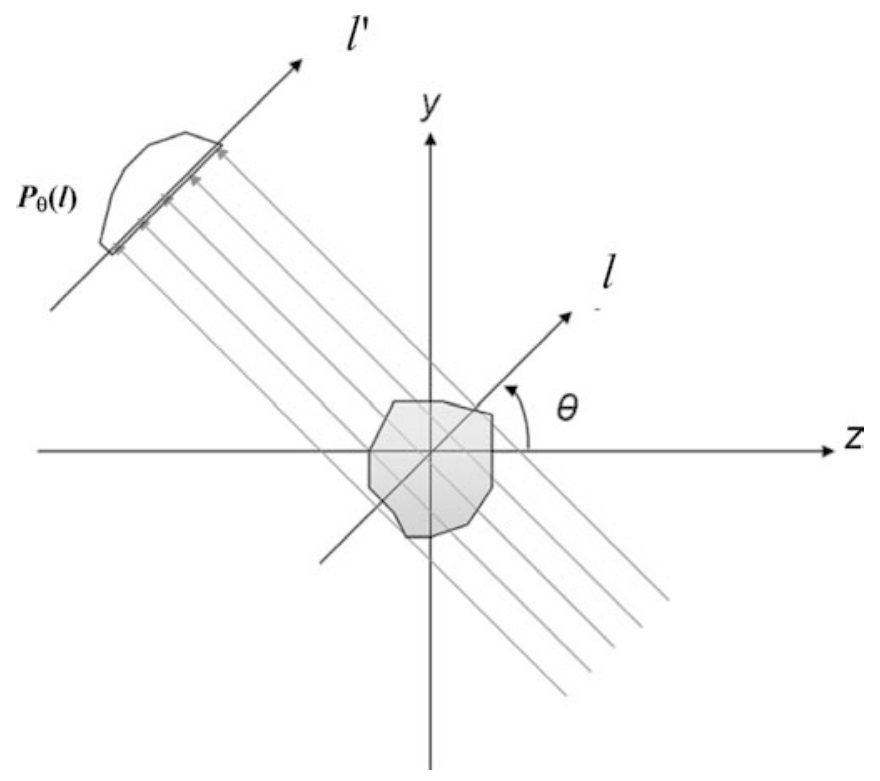

Fig. 2. Configuration of projection in one view direction at $\theta$ (Feng et al 2001) called tomography can determine two-dimensional density fields from one-dimensional projected data sets. The test field for asymmetric or generally 3-D situations must be interrogated from many different directions, yielding many projections that must be simultaneously deconvoluted. Stacking up these two-dimensional conversions will create a fully three-dimensional image from the projected two-dimensional information. Use of the optical tomography technique in flow-visualization can be traced back over three decades (Matulka and Collins 1971). Progress in reconstruction methods (Hertz 1986; Faris and Byer 1987; Cha and Sun 1989) has aided in new applications of the technique. The most difficult problem in optical tomography is the lack of sufficient data for reconstruction, which occurs when the views are truncated or inadequate in number (for non-axisymmetric three-dimensional flows).

The two most popular methods for performing this transform involve either Fourier domain (Fourier Convolution) methods or the algebraic solution to a non-unique system of equations, termed the Algebraic Reconstruction Technique (ART) (Muralidhar 2000). The Fourier domain method implemented as a convolution back projection method is simple, but suffers from reduced reconstruction accuracy when the amount of projection data is limited or restricted.

If the experimental test field is axisymmetric, only one projection is required and hence the convolution is greatly simplified and termed the Abel conversion (Walsh and Kihm 1995). While algebraic reconstruction methods are iterative and work better in cases of limited projections, a major disadvantage is that they are time-consuming, and the errors for distributions may increase after a certain number of iterations, while the errors for projections keep on reducing.

Although the Abel conversion seems suited for the data in the present work, it is highly susceptible to noise, in addition to being unsuitable for non-axisymmetric flows. This is the case with all back projection methods, as back projection alone results in a blurred reconstruction image. This is because a pure back projection adds a $1 / r$ 


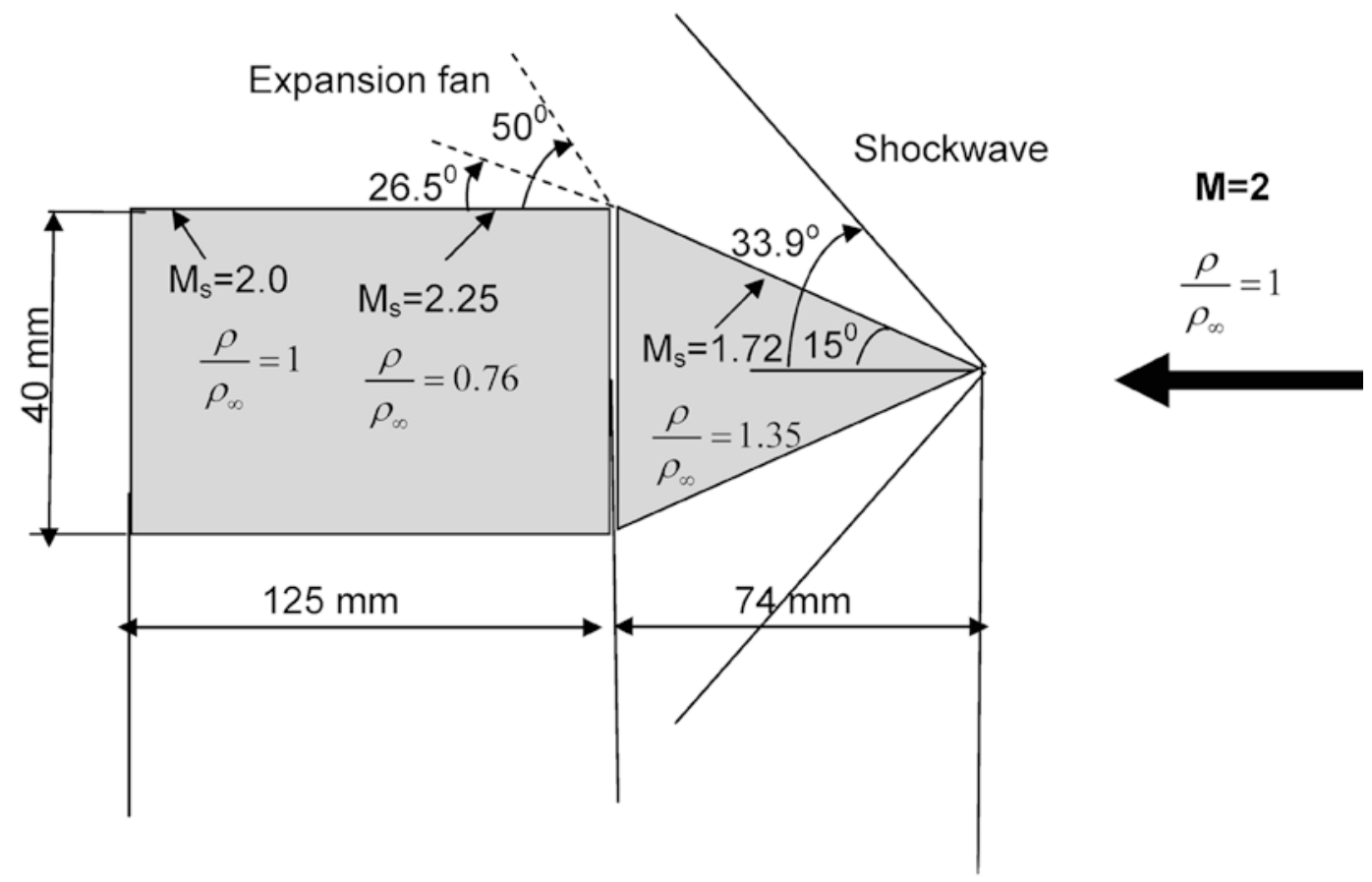

b

\section{Light Source}

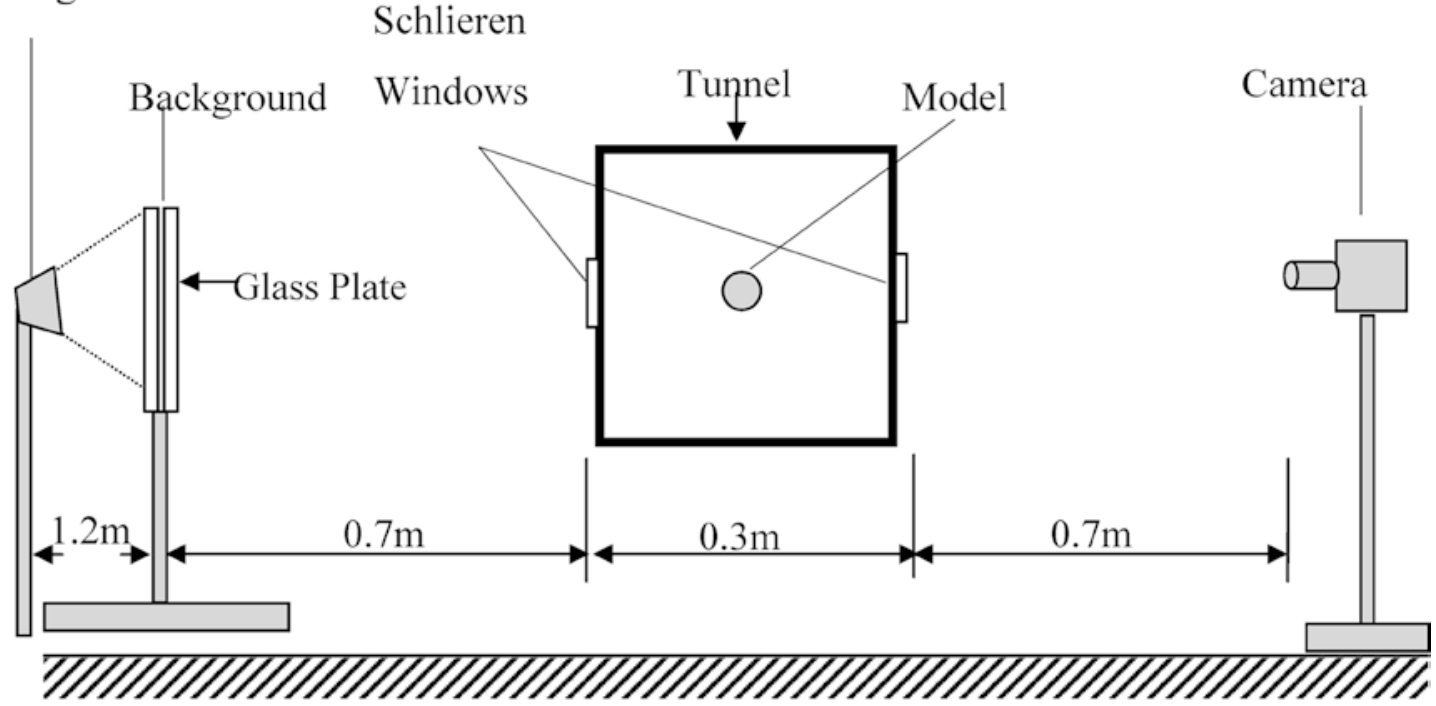

Fig. 3. a Experimental geometry of a cone-cylinder in the $0.3 \mathrm{~m}$ tunnel. b Schematic of experimental set-up

convolution to the image (Ramachandran and Lakshminarayanan 1971). Filtering must be applied to correct for this, and obtain an accurate image of the object. There are a number of choices in the type of filter to use. The simplest and most mathematically rigorous one is the ramp filter:

$H(\omega)=\pi|\omega|$

However, this filter is very sensitive to noise. For this reason, other filters are chosen for practical applications. The most commonly used filter is the Shepp-Logan filter, which combines a sinc function (which is the continuous inverse Fourier transform of a rectangular pulse of width $2 \pi$ and height unity) with the ramp filter (Shepp and Logan 1974):
$H(\omega)=\left\{\begin{array}{c}\pi|\omega| \sin c\left(\omega / \omega_{\max }\right)|\omega| \leqslant \omega_{\max } \\ 0|\omega| \leqslant \omega_{\max }\end{array}\right.$

This filter results in a small amount of blurring, but is much less sensitive to noise.

Therefore, a filtered back projection (FBP) algorithm has been used with a Shepp-Logan filter, which in addition to noise suppression makes the method suitable for both axisymmetric and non-axisymmetric flows.

This algorithm reconstructs an image from parallel beam projections. In parallel beam geometry, each projection is formed by combining a set of line integrals through an image at a specific angle. An approximation to the image is then formed based on the projections. 
In FBP, a planar cross-section $(x, y)$ (see Fig. 2) at a particular $z$ is used. For one view direction at $\theta(\theta=0$ is the $z$-axis), the projection $P_{\theta}(l)$ is calculated from the displacements obtained from the correlated images (Feng et al 2001):

$P_{\theta}(l)=\frac{n \lambda}{K}=\int_{\text {ray }} \Delta \rho(x, y) \mathrm{d} l$

Here, $l=x \cos \theta+y \sin \theta$ (see Fig. 2)

$P_{\theta}(l)=\int_{-\infty}^{\infty} \int_{-\infty}^{\infty} \Delta \rho(x, y) \delta(x \cos \theta+y \sin \theta-l) \mathrm{d} x \mathrm{~d} y$

Its Fourier transform is

$$
\begin{aligned}
S_{\theta}(\omega) & =\int_{-\infty}^{\infty} P_{\theta}(l) \mathrm{e}^{-j \omega l} \mathrm{~d} l \\
= & \int_{-\infty}^{\infty} \int_{-\infty}^{\infty} \Delta \rho(x, y) \mathrm{e}^{-j(x \omega \cos \theta+y \omega \sin \theta)} \mathrm{d} x \mathrm{~d} y
\end{aligned}
$$

Assume

$G\left(\omega_{1}, \omega_{2}\right)=\int_{-\infty}^{\infty} \int_{-\infty}^{\infty} \Delta \rho(x, y) \mathrm{e}^{-j 2 \pi\left(\omega_{1} x+\omega_{2} y\right)} \mathrm{d} x \mathrm{~d} y$

Using the Projection Slice theorem (Radon 1917)

$S(\omega)=G(\omega \cos \theta, \omega \sin \theta)=G(\omega, \theta)$

as well as

$G(\omega, \theta+\pi)=G(-\omega, \theta)$

$h(l)=\int_{-\infty}^{\infty}|\omega| e^{j \omega l} \mathrm{~d} \omega$

where $h(l)$ is a filtering function to clip high frequency noise (described later), we get

$$
\begin{aligned}
\Delta \rho(x, y) & =\int_{-\infty}^{\infty} \int_{-\infty}^{\infty} G\left(\omega_{1}, \omega_{2}\right) \mathrm{e}^{j 2 \pi\left(\omega_{1} x+\omega_{2} y\right)} \mathrm{d} x \mathrm{~d} y \\
& =\int_{0}^{\pi} \int_{-\infty}^{\infty} S_{\theta}(\omega)|\omega| \mathrm{e}^{j \omega l} \mathrm{~d} \omega \mathrm{d} \theta
\end{aligned}
$$

That is:

$\Delta \rho(x, y) \cong \int_{0}^{\pi}\left[\int_{-\infty}^{\infty} P_{\theta}(\zeta) h(l-\zeta) \mathrm{d} \zeta\right] \mathrm{d} \omega$

The distribution of $\Delta \rho(x, y)$ at a certain $z$ level is calculated from the function $h(l)$ and the projection $P_{\theta}(l)$. Then the cross-sections can be stacked to form a 3-D distribution.

A more accurate result can be obtained by using more projections in the reconstruction. As the number of projections (equivalent to the number of views; for a steady flow, these views need not be recorded simultaneously) increases, the reconstructed image more accurately approximates the original image. In the present study however, the flow being axisymmetric, all of the projections are identical. The filtered back projection algorithm filters the projections using the Shepp-Logan filter described earlier (Eq. 5) and then reconstructs the image using the filtered projections.

In some cases, noise can be present in the projections. This noise, usually high frequency, has to be clipped without affecting the density distribution. To achieve this, a method known as reduced bandlimit for projection has been proposed and successfully used earlier for other tomographic applications (Feng et al 2001). In this method, the function $h(l)$ (Eq. 11) can be rewritten by making the bandlimited projection assumption, as follows:

$h(l)=\int_{-\infty}^{\infty} H(\omega) e^{i \omega l} d \omega$

$H(\omega)=\left\{\begin{array}{c}|\omega| \text { if }|\omega|<W \\ 0 \text { otherwise }\end{array}\right.$

where $W$ is the maximum frequency, the bandlimit, which is usually half of Nyquist. The influence of noise in the projections is assumed to belong mainly to the high frequency in the Fourier domain. The bandlimit, $W$, is searched for in each projection in the Fourier domain. $W$ is determined to be where the filtered projection $\left[h * P_{\theta}\right]$ has least fluctuation. It works like a low-pass frequency filter. On the other hand, the bandlimit $W$ should be as large as possible. Therefore, every projection (this test case being axisymmetric, a single projection suffices) is assigned a different reduced bandlimit, so it is termed the "reduced bandlimit technique". Further details and the validation of the reduced bandlimit technique can be found in Feng et al (2001). During processing, a Hamming window is employed for smoothing. The Hamming window helps to avoid discontinuities at the ends, tapering the signal to zero or near zero and therefore reducing the mismatch, and is defined as (Oppenheim and Schafer 1989):

$\omega_{n}=\left\{\begin{array}{c}0.54-0.46 \cos (2 \pi n /(N-1)) 0 \leqslant n<N \\ 0 \text { otherwise }\end{array}\right.$

where $\omega_{n}$ is the frequency at the $n^{\text {th }}$ point in the time series. Therefore, the influence of noise can be reduced by neglecting the high frequency, while the density distribution may not be affected.

The implementation of this method has been done using the inverse Radon transform, which is well documented in literature (Kak and Slaney 1988).

\section{4}

\section{Experiments}

The experiments were carried out in the $0.3 \mathrm{~m} \times 0.3 \mathrm{~m}$ supersonic test section of the trisonic wind tunnel at NAL. In this tunnel, supersonic Mach numbers are produced using fixed nozzle block liners. The model configuration was a $15^{\circ}$ (semi-apex angle) cone-cylinder. This was chosen because the density field behind the conical shock wave at zero incidence, available from cone tables (Sims 1964), can be utilized for comparison with the results obtained by BOS. The model details are given in Fig. $3 \mathrm{a}$. 


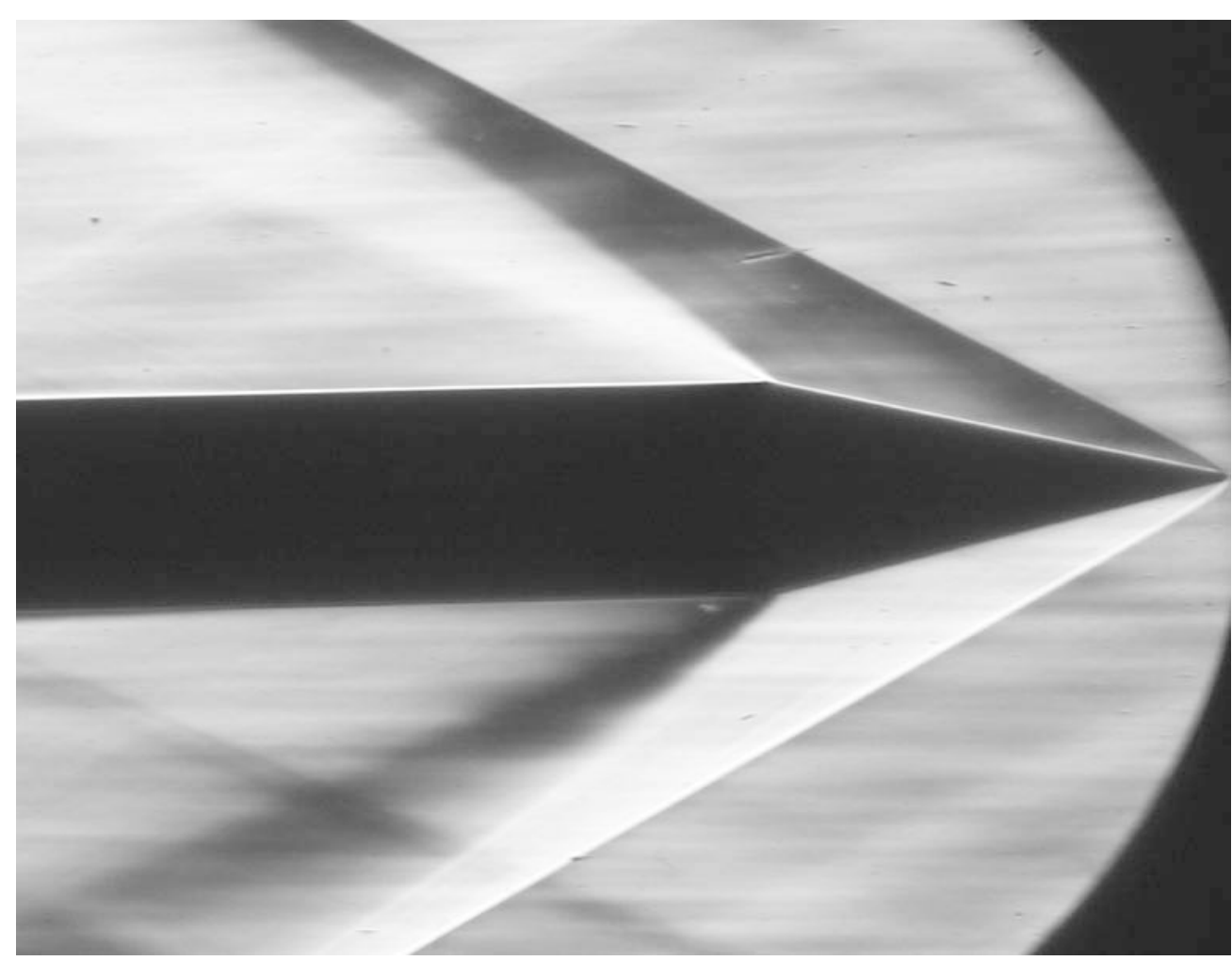

Fig. 4. Conventional Schlieren (horizontal knife-edge) of the flow over a $15^{\circ}$ cone-cylinder at Mach 2.0 in the $0.3 \mathrm{~m}$ trisonic tunnel
Tests were made at a Mach number of 2.0. The settling chamber total pressure was $2.1 \mathrm{~kg} / \mathrm{cm}^{2}$ and the Reynolds number based on cylinder diameter $(D=0.04 \mathrm{~m})$ was $2 \times 10^{4}$. Some of the broad features of the flow, like the shockwave angle, the features of expansion fan, and surface Mach numbers are included as well in Fig. 3a.

For the BOS, a structured background to focus on was created by means of a normal random number generator. This generated a matrix, $2000 \times 2000$ in size, of random numbers whose elements were normally distributed with zero mean, unit variance and standard deviation. The matrix was plotted as a binary image - black dots for all non-zero values. A normal distribution was chosen to minimize the errors away from the optical axis. Since vibrations often present in the wind tunnel environment can cause erroneous results on cross-correlation, care should be taken to minimize vibrations of the background and camera. Further, choosing a large enough exposure time (here $1 / 100^{\text {th }}$ of a second) and averaging several displacement fields can successfully address this problem. The printed out pattern was sandwiched between two thin sheets of glass and mounted on a heavy stand fixed on a concrete block to minimize vibrations (see Fig. 3b). It was then illuminated to achieve a better signal-to-noise ratio by placing a large halogen lamp far enough behind so as to approximate parallel beam conditions, eliminating the need for methods to re-sort sets of fan beam projections into parallel beam projections (Kak and Slaney 1988).

The optimal location for the background, light source and camera were arrived at by using Eq. 3 and keeping in mind that increasing sensitivity (displacement of image) meant lower physical resolution, as the interrogation size used in the correlation algorithm would have to be correspondingly larger.
By using the Schlieren windows built into the tunnel, we could get the direction of viewing to be perpendicular to the background and through the flow. The windows were made of optical quality $(\lambda / 8)$ glass and did not introduce any distortions. The imaging was achieved by means of a commercially available Sony DSC F-707 digital still camera with 5.1 megapixel resolution. The camera was mounted on a heavy tripod, fixed to the ground, $0.7 \mathrm{~m}$ away from the tunnel. Figures $4 \mathrm{a}, \mathrm{b}$ show the background dot pattern imaged with the cone-cylinder in the foreground in the absence and presence of the flow respectively. They appear identical due to the high density of the dots in the background image. Eight such image sets were taken both with and without the flow. The displacements calculated from these data sets were then averaged to eliminate any noise caused by vibration, to obtain the output vector field as described in the next section.

\section{5}

\section{Image analysis}

The images were analyzed by means of a cross-correlation algorithm developed for the purpose. The sub-pixel accuracy of the calculated displacement was 0.1 pixels using a Gaussian sub-pixel interpolation technique commonly used in Particle Image Velocimetry. The maximum displacement for the present optical setup was found to be 3 pixels, and so an interrogation window of $16 \times 16$ pixels was found to be more than adequate; this translated into a physical resolution of $2 \mathrm{~mm}$. The output data was plotted on a $160 \times 160$ rectangular mesh. Figure 5 shows the average (of eight images) displacements as a vector field, the magnitude and direction of which are the density gradients of the flow (the arrows point towards lower density). 

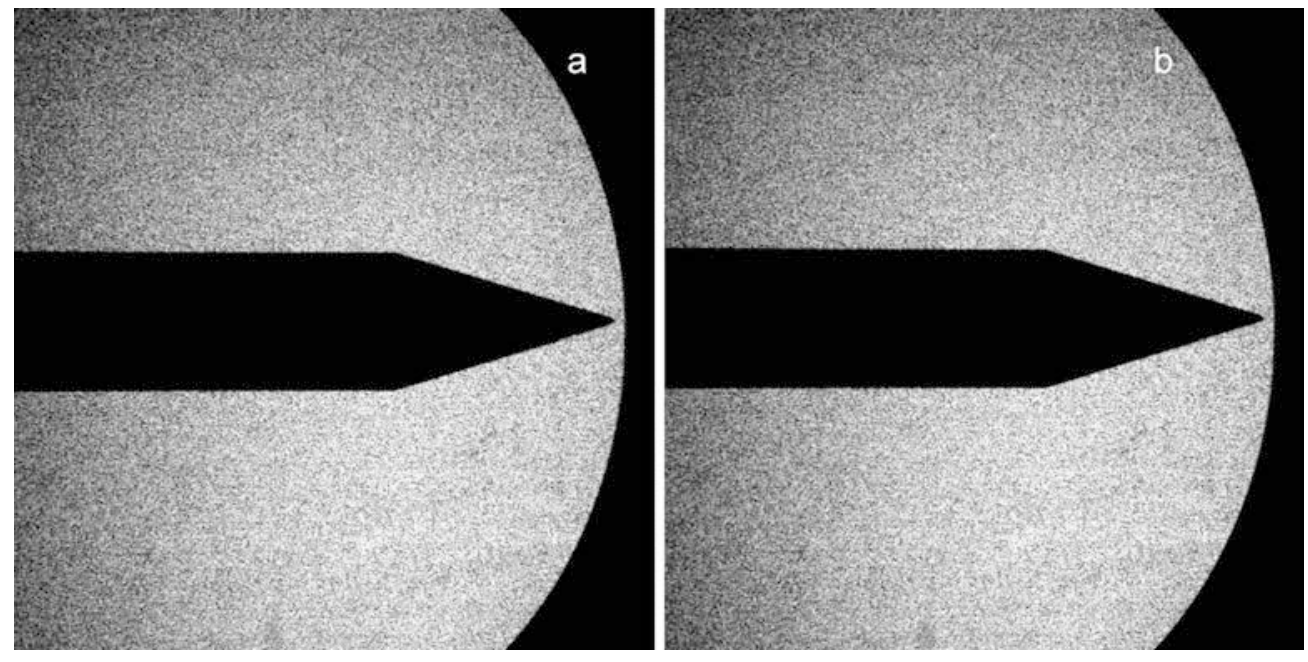

Fig. 5. The background dot pattern a without the flow and b with the flow
The derivative of the density gradients forms an elliptic partial differential equation of the form:

$\frac{\partial^{2}}{\partial x^{2}} \rho(x, y)+\frac{\partial^{2}}{\partial y^{2}} \rho(x, y)=S(x, y)$

so $\nabla^{2} \rho=S$

where $S(x, y)$ is the source term, which is calculated at each mesh point from the displacements obtained through correlation. Equation 17 is also commonly known as the Poisson equation. Neumann boundary conditions are chosen in which normal derivative $\hat{n} \cdot \vec{\nabla} \rho$, $\hat{n}$ being the outward normal to the surface is specified at the boundaries. This implies that the values of $\frac{\partial \rho}{\partial x}$ and $\frac{\partial \rho}{\partial y}$ are specified at each mesh point on the mesh boundary parallel to the $y$ - and $x$-axes respectively. This is solved on the same rectangular mesh using the Successive Over Relaxation method (Ehrlich 1981) as above, and yields the line-ofsight integrated density field, shown in Fig. 6.

This density field is then used as the projected data set for the filtered back projection. As seen before, since this flow is axisymmetric it requires only a single data set and the viewing angle $(\theta)$ is therefore set to zero. The contribution to the noise is largely from the limitations of image resolution and is assumed to belong mainly to high frequency in the Fourier domain (Feng et al 2001). This is minimized using the Shepp-Logan filter discussed earlier.

\section{6}

\section{Results and discussion}

Figure 7 shows the back-projected normalized density field at one viewing angle. The value of the freestream total density $\left(\rho_{\infty}\right)$ is used for normalization. The regions of low

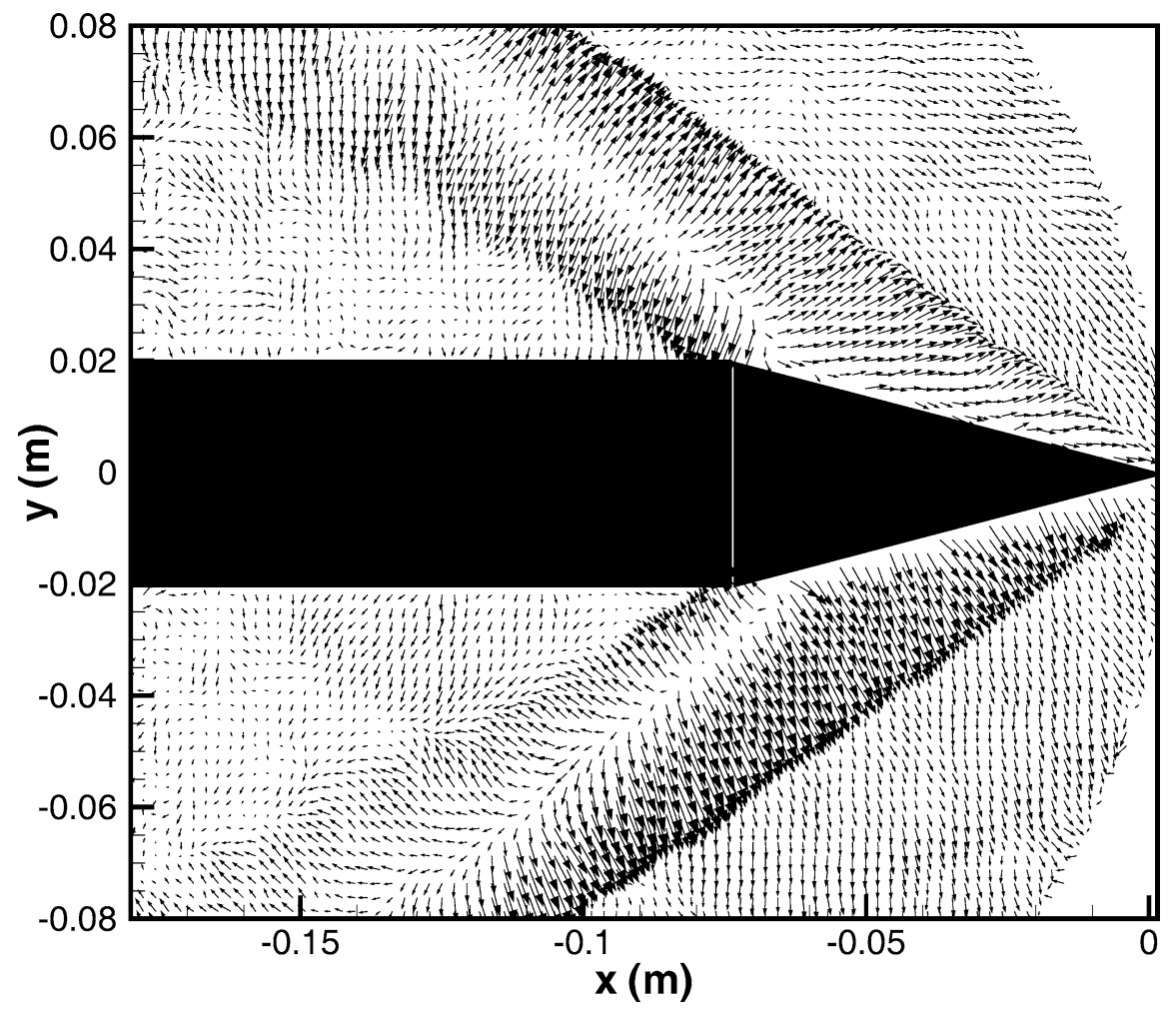

Fig. 6. The average displacement field: vectors of density gradients 


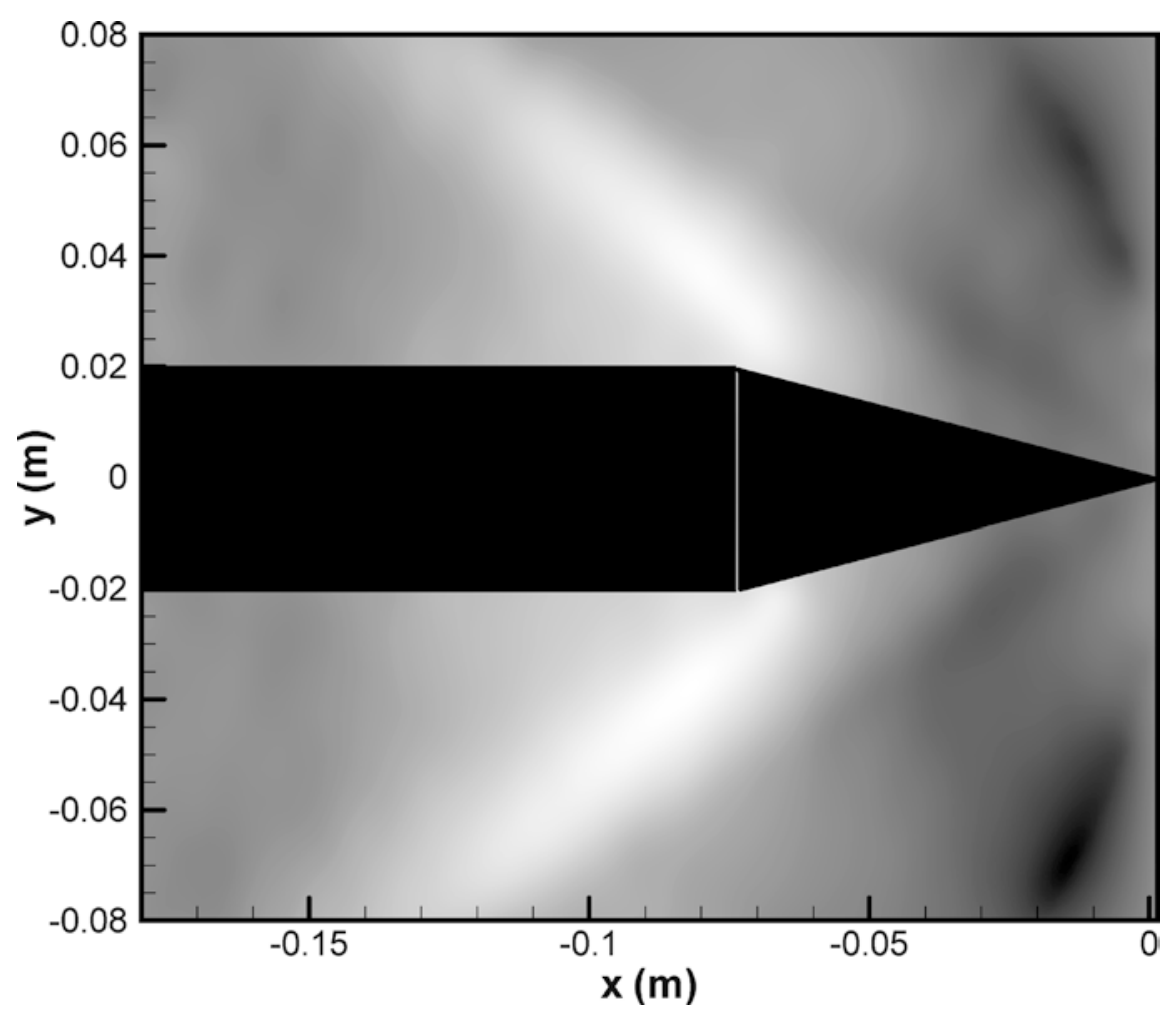

Fig. 7. The projected density field, as computed from the Poisson equation and high density ratios corresponding to the shock and expansion fan can be clearly seen. The areas of low density ratios downstream of the Prandtl-Meyer expansion are due to the overexpansion effect. As this is followed by a recompression effect, the density ratio is seen to return to the freestream value. The slight patchiness observed in the freestream upstream of the shock is probably due to a slight vibration of the background. Averaging of the displacement fields ensured no directional density gradients in such regions, but resulted in slight noise. No attempt has been made to filter out the noise from the entire region, to avoid artifacts generated by the filtering process. However the signal-to-noise ratio in the regions of interest is adequate for drawing unambiguous conclusions.

In order to determine the integration constant in Eq. 2, we need to know and match to a reference density at one point in the field. In this application, the freestream total density $\left(\rho_{\infty}\right)$ for the chosen Mach number is easily calculated using isentropic relations, as the tunnel settling chamber pressure and temperature are known. This value in the freestream is used as the reference and determines the constant. The freestream total density $\left(\rho_{\infty}\right)$ is also used to normalize the density field. The variation of the density ratio $\left(\frac{\rho}{\rho_{\infty}}\right)$ at a streamwise location of $20 \mathrm{~mm}$ from the cone apex, as a function of angle ( $\beta$ ) from the cone surface to the shock is plotted in Fig. 8. Again from isentropic relations, the shockwave angle ( $\left.\beta_{\text {shockwave }}\right)$ is found to be $33.9^{\circ}$.The computed values at the corresponding location from cone tables (Sims 1964) are also plotted in the figure for comparison. The agreement may be seen to be very good considering the resolution of the interrogation window $(2 \mathrm{~mm})$ used for the calculation of density gradients.

Figure 9 shows density variations downstream of the expansion fan at two streamwise locations of $2.5 \mathrm{D}$ $(\cong 0.10 \mathrm{~m})$ and $5.0 \mathrm{D}(\cong 0.20 \mathrm{~m})$ from the cone apex. The density at the surface, as calculated from isentropic calculations, is also shown for comparison with BOS. The expansion fan at the cone-cylinder junction is locally twodimensional and leads to higher Mach number (compared to the freestream value $M_{\infty}$ ) immediately downstream, as has also been reported by other studies (Coustols and Cousteix 1994). As the flow proceeds downstream along the cylinder, further recompression takes place, resulting in a value close to $M_{\infty}$. It was not possible to obtain density values closer than $5 \mathrm{~mm}$ from the cylinder surface because of the limitation on the measurement resolution and the fact that it was very close to the expansion fan at the junction. Downstream $(x=5.0 d)$ of the recompression waves, the flow has returned to near freestream values, and the profiles show the expected pressure loss.

From the density field, we can obtain either Schlieren or shadowgraph images by computing the first or second derivative in the direction required (knife-edge vertical or horizontal). Here, the vertical gradient (horizontal knifeedge) was chosen, as was the case in the conventional Schlieren (Fig. 10). Figure 11 shows the computed Schlieren image. Figure 10 may be referred to for a comparison. It can be seen that the shock and its interaction with the expansion has been captured, and the wave angles are as expected. Despite the noise due to the mathematical operation of computing the derivatives, the weaker reflected shock (from the boundary) has also been captured. However, the width of the shock is larger than that in the conventional Schlieren, because of the resolution chosen for BOS image analysis $(2 \mathrm{~mm})$. The shock region can be made thinner in BOS images if a higher resolution cross-correlation algorithm is chosen for the interrogation of images.

The effect of the camera resolution and sensitivity (bits/pixel) was also assessed on the density field 


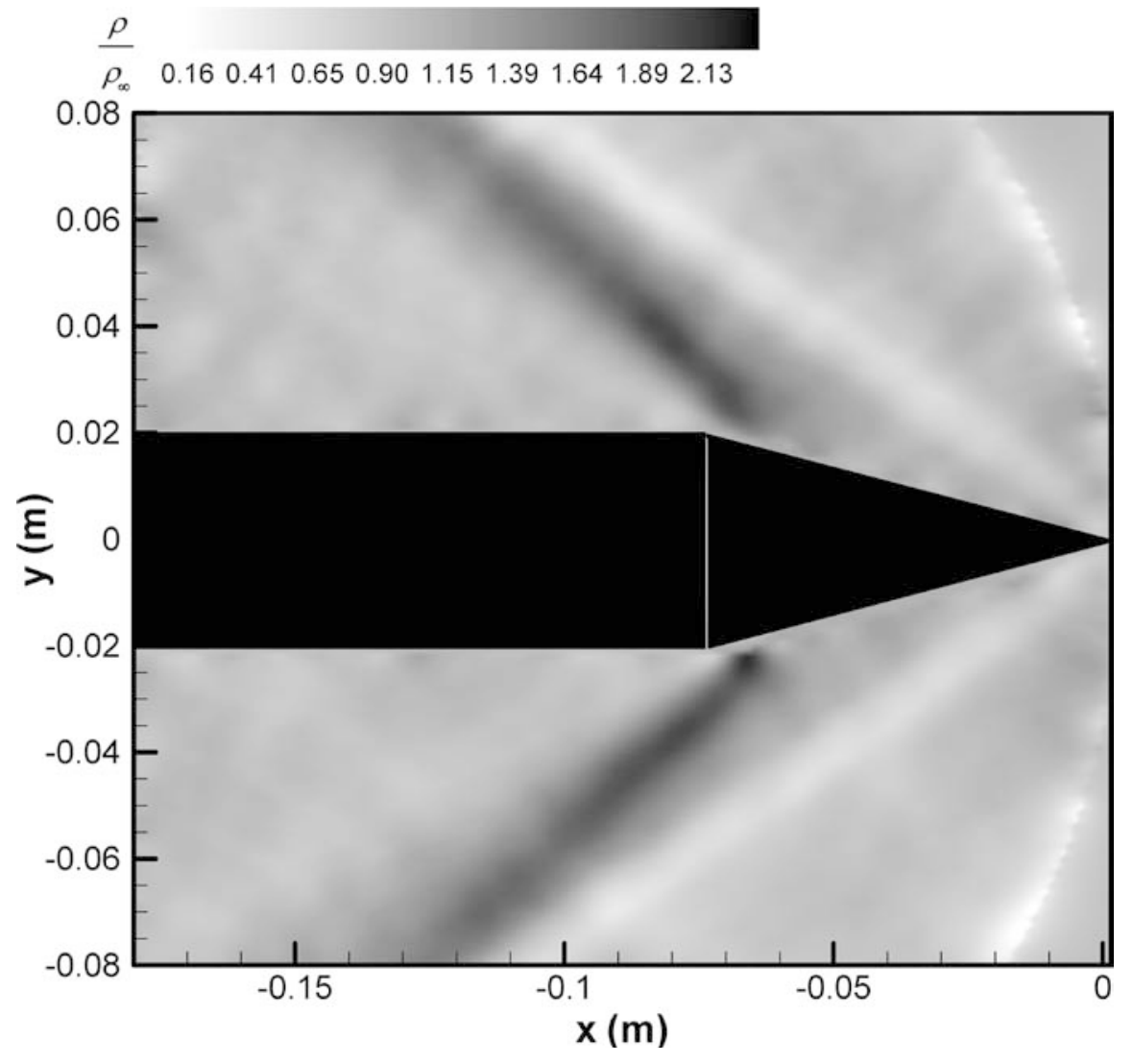

calculated by BOS. For this purpose a scientific grade Kodak ES 1.0 cross-correlation camera (normally used for PIV measurements) with a 1.0 megapixel was also used in the present experiment for identical flow conditions. It was found that the SONY digital camera (with 5.1 megapixel resolution) provided significantly

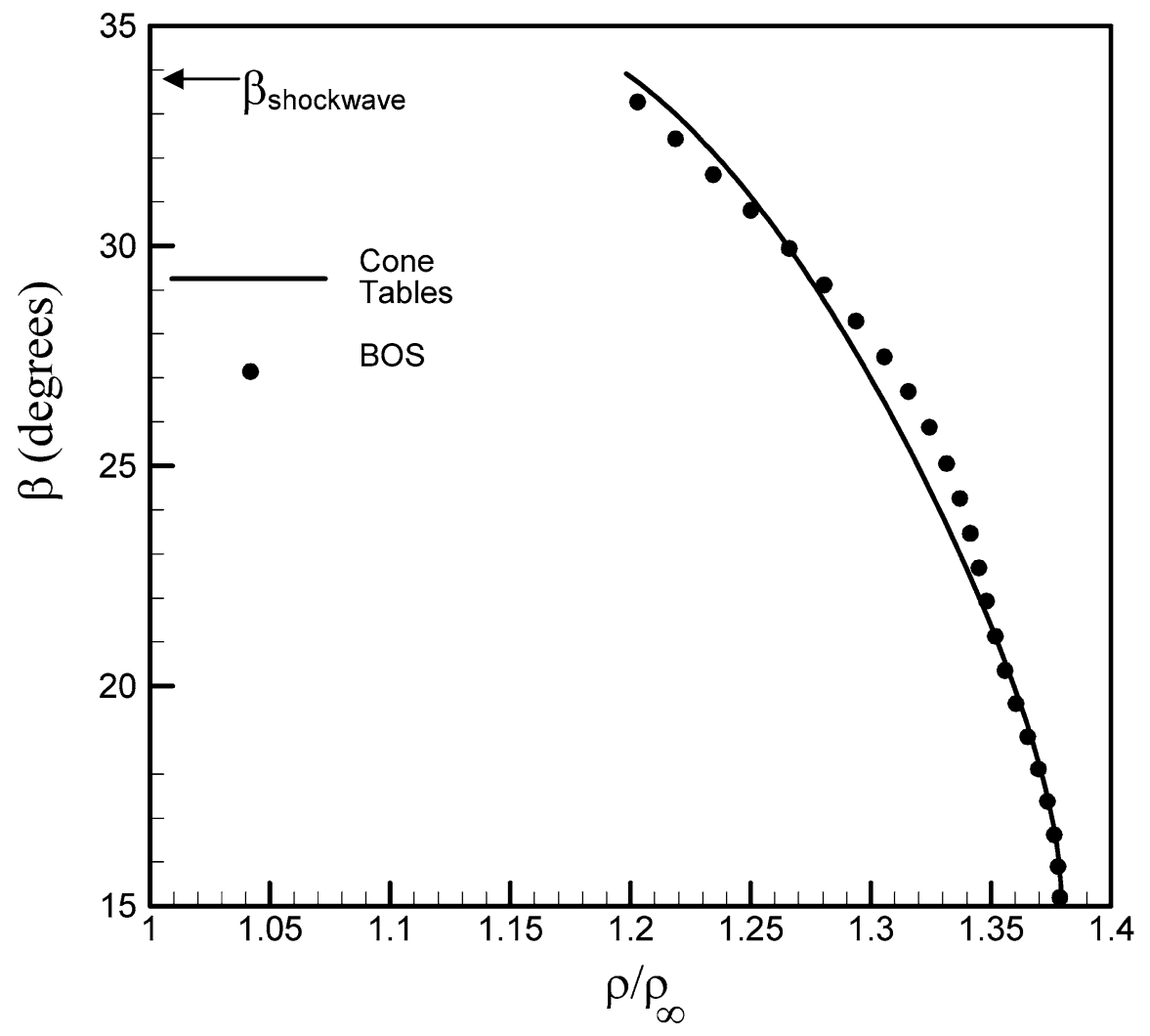

Fig. 8. The back-projected normalized density field, as viewed from one direction 


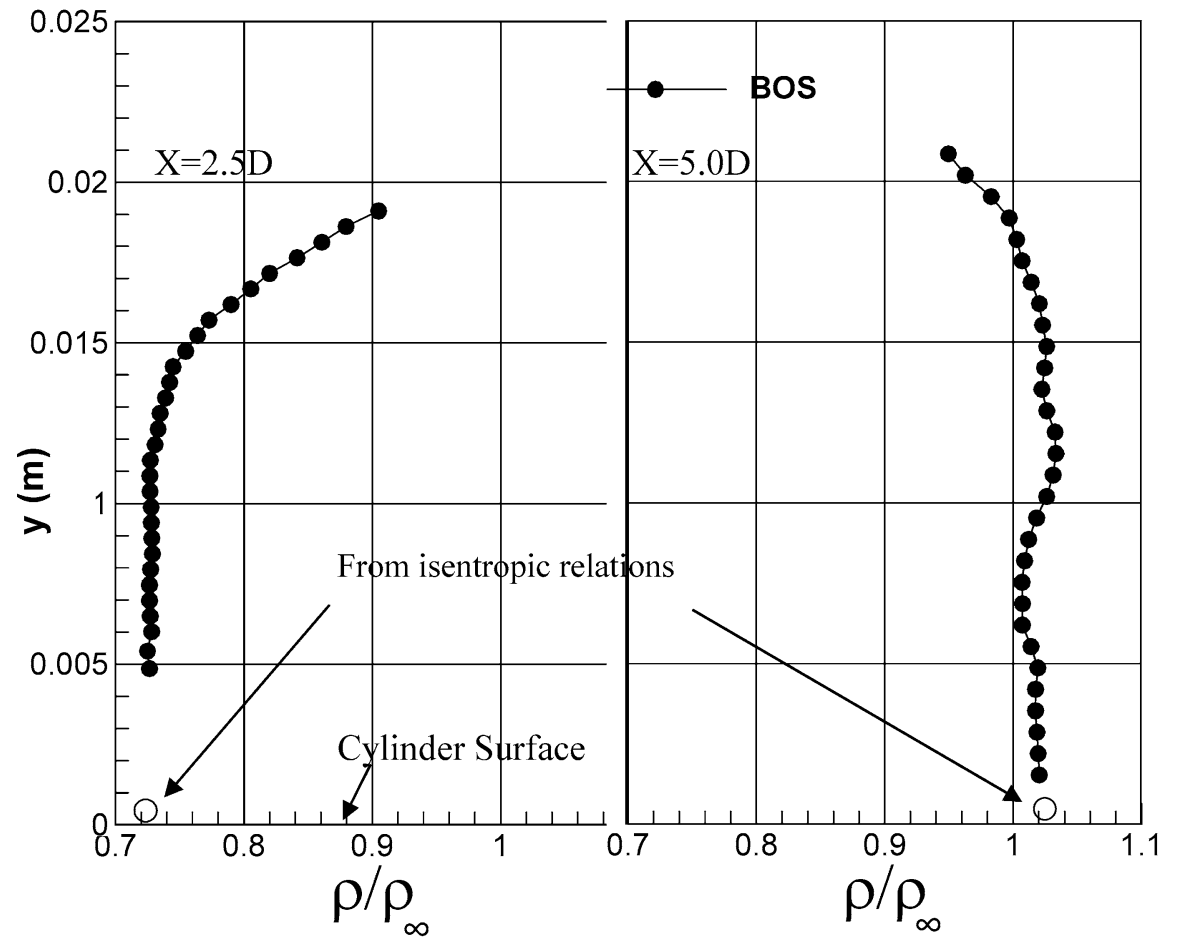

Fig. 10. Density profiles at different streamwise locations enhanced image details, indicating the importance of a high-resolution camera for the BOS technique. Most off-the-shelf digital still cameras nowadays offer such resolution, which benefits the general application of this technique.

\section{7}

\section{Conclusions}

An investigation was carried out to verify the density field determined using a BOS technique for the first time. The experimental configuration chosen was an axisymmetric cone-cylinder in a Mach 2.0 flow. The BOS technique has been successfully validated by comparing the results with data from cone tables and isentropic solutions. The software analysis used involves determination of displacements using cross-correlation, calculation of the integrated line-of-sight density field from the Poisson equation, and optical tomography in one plane, employing Filtered Back Projection technique. While the size and pixel count of the CCD limits physical resolution, usage of higher resolution CCDs would result in lower noise, without sacrificing imaged area.

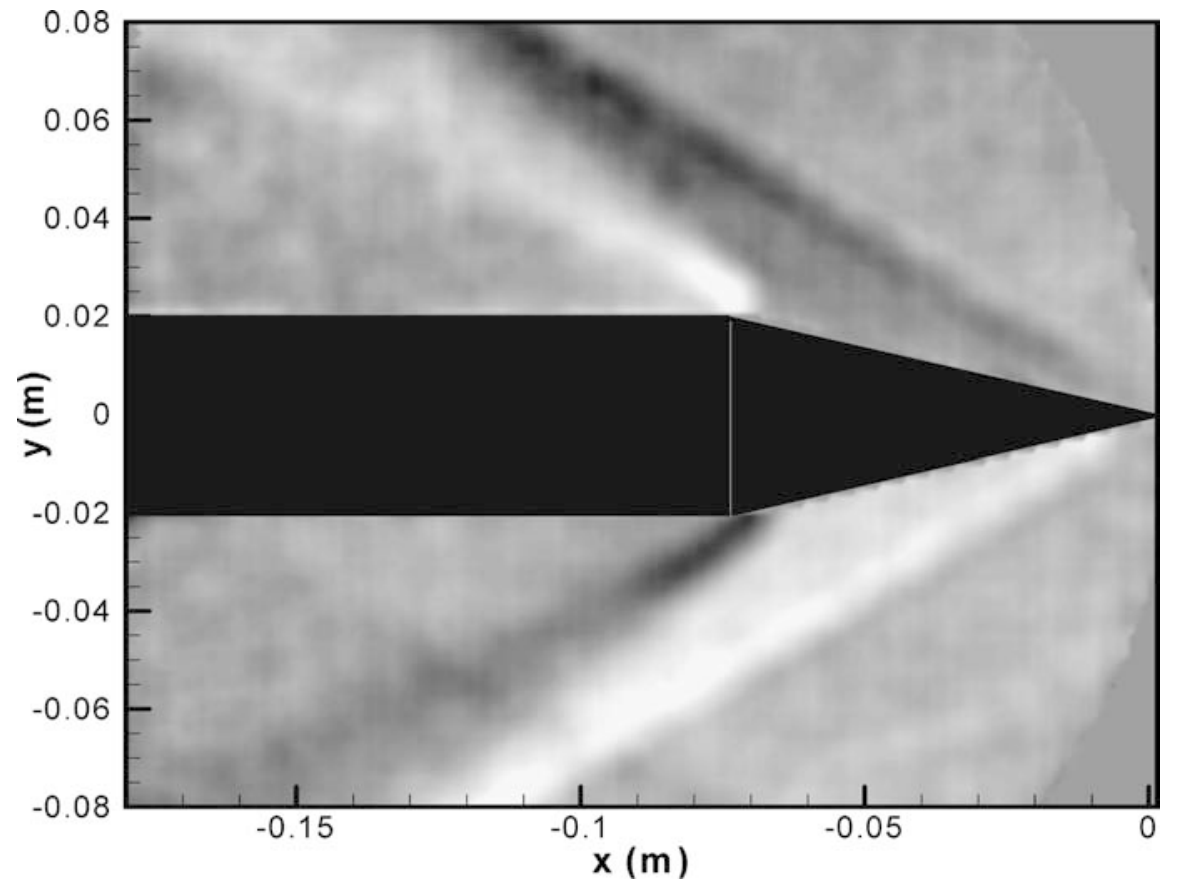

Fig. 11. Computed Schlieren (horizontal knife-edge) 
While the Abel transform is often used in the context of axisymmetric flow fields, recent studies have shown that it is susceptible to noise in the projected data sets. The filtered back-projection technique is seen to workvery well at minimizing the effects of the noise inherent in such imaging and the calculation of small particle displacements. In addition, the technique is not limited to twodimensional flows and can be implemented on non-axisymmetric flows using multiple view angles.

\section{References}

Cha SS, Sun H (1989) Interfereometric tomography of continuous fields with incomplete projections. Opt Lett 14(6):299-301

Coustols E, Cousteix J (1994) Performance of riblets in the supersonic regime. AIAA J 32:431-433

Dalziel SB, Hughes GO, Sutherland BR (1998) Synthetic Schlieren. In: Carlomagno GM (ed) Proc $8^{\text {th }}$ Int Symp Flow Visualization, Sorrento, Italy, 1-4 September 1998, ISBN 0953399109 (CDROM)

Dalziel SB, Hughes GO, Sutherland BR (2000) Whole field density measurements by 'synthetic Schlieren'. Exp Fluids 28:322-335

Ehrlich L (1981) An ad-hoc SOR method. J Comput Phys 43:31-45

Faris W, Byer RL (1987) Quantitative three-dimensional optical tomographic imaging of supersonic flows. Science 238:1700-1702

Feng J, Okamoto K, Tsuru D, Madarame H, Fumizawa M (2001) Visualization of 3D gas density distribution using optical tomography. Chem Eng J 3865:1-9

Goldstein RJ (1983) Fluid mechanics measurements. Hemisphere, Washington, DC

Hertz HM (1986) Kerr effect tomography for non-intrusive spatially resolved measurements of asymmetric field distribution. Appl Opt 25:914-921

Kak AC, Slaney M (1988) Principles of computerized tomographic imaging. IEEE, New York

Matulka RD, Collins DJ (1971) Determination of three-dimensional density fields from holographic interferograms. J Appl Phys 42(3):1109-1119
McMackin L, Masson B, Clark N, Bishop K, Pierson R, Chen E (1995) Hartmann wave-front sensor studies of dynamic organized structure in flowfields. AIAA J 33:2158-2164

Meier GEA (1999) New optical tools for fluid mechanics. In: Proc $8^{\text {th }}$ Int Symp Flow Visualization, Sorrento, Italy, 1-4 September 1998, ISBN 0953399109 (CD-ROM), Paper 226

Meier GEA (2002) Computerized background oriented Schlieren. Exp Fluids 33:181-187

Merzkirch W (1987) Flow visualization. Academic, New York

Muralidhar K (2000) Temperature field measurement in buoyancy driven flows using interferometric tomography. Ann Rev Heat Transfer 12:265-375

Onu K, Flynn MR, Sutherland BR (2003) Schlieren measurement of axisymmetric wave amplitudes. Exp Fluids 35:24-31

Oppenheim AV, Schafer RW (1989) Discrete-time signal processing. Prentice-Hall, Englewood Cliffs, NJ, pp 447-448

Raffel M, Richard H, Meier GEA (2000) On the applicability of background oriented optical tomography for large scale aerodynamic investigations. Exp Fluids 28:477-481

Radon J (1917) On the determination of functions from their integrals along certain manifolds. Ber Saechsische Akad Wiss 29:262-277

Ramachandran GN, Lakshminarayanan AV (1971) 3D reconstructions from radiographs and electron micrographs: Application of convolution instead of Fourier transforms. P Natl Acad Sci 68:2236-40

Richard H, Raffel M, Rein M, Kompenhans J, Meier GEA (2000) Demonstration of the applicability of a Background Oriented Schlieren (BOS) Method. In: Adrian RJ (ed) Proc $10^{\text {th }}$ Int Symp Appl Laser Techniques to Fluid Mechanics, Lisbon, Portugal, 1013 July 2000, CD-ROM

Sims JL (1964) Tables for supersonic flow around right circular cones at zero angle of attack. NASA SP-3004

Shepp LA, Logan BF (1974) The Fourier reconstruction of a head section. IEEE T Nucl Sci NS-21:21-43

Sutherland BR, Dalziel SB, Hughes GO, Linden PF (1999) Visualization and measurement of internal gravity waves by 'Synthetic Schlieren', part I: Vertically oscillating cylinder. J Fluid Mech 390:93-126

Walsh TE, Kihm KD (1995) Tomographic deconvolution of laser speckle photography for axisymmetric flame temperature measurement. J Flow Vis Image Process 2:299-310 